\title{
Aplicación del Modelo Van Hiele Para la Enseñanza de la Geometría Analítica
}

Ruth M. González S. ${ }^{1}$

\section{Resumen}

No cabe duda que el estudio de los temas vinculados a la geometría lleva intrínsecas múltiples dificultades, cuyo análisis y caracterización ha sido objeto de estudio en muchos ámbitos educativos, encontrándose que algunas dificultades específicas vinculadas con el lenguaje de geometría que están vinculadas con la lectura y comprensión de palabras. Nuestro lenguaje verbal diario posee muchos términos geométricos básicos que nos permite comunicarnos y entendernos con mayor precisión acerca de observaciones sobre el mundo en que vivimos. Es por esto que algunos estudiantes no alcanzan el rendimiento esperado en la asignatura, generando así en ellos problemas de repitencia y deserción académica. La presente investigación muestra una ayuda para interpretar la evolución del razonamiento geométrico en los estudiantes y lo constituye el modelo de desarrollo del razonamiento geométrico elaborado por los esposos Van Hiele. Cuyos componentes principales son la "teoría de los niveles de razonamiento", que explica cómo se produce el desarrollo en la calidad de razonamiento geométrico de los estudiantes cuando éstos estudian geometría, y las "fases de aprendizaje", que constituye su propuesta didáctica para la secuenciación de actividades de enseñanza-aprendizaje en el aula, con el objeto de facilitar el ascenso de los estudiantes de un nivel de razonamiento al inmediatamente superior. De los resultados encontrados se resalta la idea que el estudiante que ingresa al primer semestre de la licenciatura en matemática e informática, se encuentra con muchas dificultades en conceptos básicos de geometría lo que impide la adquisición del nuevo conocimiento. Se encontró que el modelo puede ser aplicado en cualquier tópico de conocimiento matemático ya que es flexible, garantizando así a los estudiantes una forma valida de aprender significativamente procesos geométricos para rendir de manera satisfactoria en la Geometría Analítica

Palabras Clave: Modelo, Reconocimiento, Clasificación, Deducción o demostración, Rigor, Recursividad, secuencialidad, Especificidad

\section{Abstract}

There is no doubt that the study of issues related to the intrinsic geometry has multiple difficulties, analysis and characterization which has been studied in many educational settings, finding that some specific difficulties associated with the language of geometry that are linked with reading and understanding of words. Our daily oral language has many basic geometric terms allows us to communicate and understand with greater precision observations about the world in which we live. That is why some students do not achieve the expected performance in the subject, so generating them and dropout issues academic. This research shows an aid to interpreting the evolution of geometric

${ }^{1}$ Universidad Francisco de Paula Santander

ruthmery@ufps.edu.co 
reasoning in students and what is the model for development of geometric reasoning developed by Van Hiele spouses.

Whose main components are the "theory of levels of reasoning," which explains how development occurs in the quality of geometric reasoning of students as they study geometry, and the "learning phase", which is its didactic approach sequencing of the teaching and learning in the classroom, in order to facilitate the ascent of the students from one level to next higher reasoning. Of the findings highlight the idea that students entering the first semester of mathematics and computer science degree, he finds great difficulty in basic concepts of geometry which prevents the acquisition of new knowledge. It was found that the model can be applied to any topic of mathematical knowledge as it is flexible, ensuring students learn a valid form of geometric processes to yield significantly satisfactorily in Analytic Geometry.

Key words: Model, Recognition, Classification, deduction or demonstration, Rigor, Recursion, sequence, specificity

\section{Introducción}

$\mathrm{R}$ ecientes investigaciones destinadas a evaluar la enseñanza de la matemática evidencian el estado critico en el que esta se encuentra, destacándose como indicadores el bajo rendimiento académico a nivel general en dicha asignatura y la deficiente preparación preuniversitaria, de un alto porcentaje de estudiantes que ingresan a la Universidad, en todas las áreas que abarca la matemáticas. Esto indica que las metodologías aplicadas en la enseñanza de la matemática y específicamente la geometría, no están siendo las más adecuadas puesto que cada área requiere de un razonamiento distinto para su estudio debido a las notables diferencias que hay entre ellas. (Ronny Wilson, Marianella Alpizar,2007-2009). Tal y como lo señala Bressan (2000), una de las preguntas que se debe hacer el docente es $i$ Qué tipo de problemas hemos de presentar a los estudiantes para que su actividad e investigación en torno a los mismos les conduzca hacia formas superiores de intuición y abstracción geométrica? La geometría ayuda a estimular ejercitar habilidades de pensamiento y estrategias de resolución de problemas. Da oportunidades para observar, comparar, medir, conjeturar, imaginar, crear, generalizar y deducir. Tales oportunidades pueden ayudar al estudiante a aprender cómo descubrir relaciones por ellos mismos y tornarse mejores solucionadores de problemas. La intención de esta investigación es evaluar la aplicación del modelo propuesto por la Teoría Van Hiele en la asignatura Geometría analítica, específicamente en los tópicos de "ángulo entre rectas y distancia de un punto a una recta", teniendo en cuenta el nivel de razonamiento geométrico que alcanzará un grupo de estudiantes pertenecientes al programa de Licenciatura en Matemáticas e Informática de la Universidad Francisco de Paula Santander una vez cursando dicha asignatura.

¿Dónde radican las causas de las insuficiencias en el aprendizaje de la geometría y el desarrollo del pensamiento geométrico? Algunos de los aspectos para dar respuesta a esta pregunta es que el diseño de los diferentes componentes del proceso de enseñanza aprendizaje no se adecua al contexto, al no tener en cuenta los resultados del diagnostico integral y en su gran mayoría siguen empleándose los estilos tradicionales de concepción de los sistemas de clase. Existe un inadecuado tratamiento didáctico metodológico de los contenidos, la formación de conceptos se hace de manera rutinaria y Los problemas que se proponen no parten del contexto. Los Van Hiele (1970), en sus estudios recalcan que las formas de razonamiento geométricos de distintos nivel "no sólo se reflejan en la forma de solucionar problemas sino en la forma de expresarse y en el significado que se le da a determinado vocabulario"... De allí la necesidad de que el docente interprete el vocabulario que usan sus estudiantes, pero al mismo tiempo tienda a mejorarlo y rigorizarlo, proveyéndole de mejores herramientas para expresar sus pensamientos. Esto implica evaluar la posibilidad de introducir cambios significativos en el nivel de razonamiento geométrico, razón por la cual se pretende con la presente investigación. Con lo anteriormente expuesto queda clara la existencia de dificultades en el proceso de enseñanza-aprendizaje de los estudiantes para alcanzar un alto nivel de razonamiento geométrico. Alrededor de este tema se han desarrollado innumerables investigaciones, 
por ejemplo, Michael de Villiers (1996) ,The Future of Secondary School Geometry. La lettre de la preuve, Novembre-Décembre 1999. Investigaciones Rusas sobre la enseñanza de la geometría Traducido por Martín Acosta. Algunos desarrollos en la enseñanza de la geometría (1).La teoría de Van Hiele.. El currículo de geometría de la escuela primaria y secundaria. Gloria María Braga, Profesora del Departamento de Ciencias de la Educación de la Universidad de Oviedo, España. Artículo. "Apuntes para la enseñanza de la geometría". Articulo publicado en la revista Signos, Teorías y Practicas de la Educación. Número 4, Paginas 52 57. Julio- Diciembre de 1991, entre otros. Con la realización de esta investigación propuesta se pretende responder el siguiente interrogante:

¿La aplicación del modelo Van Hiele en la enseñanza de la Geometría Analítica produce diferencias significativas en el proceso enseñanzaaprendizaje en los estudiantes de primer semestre de Licenciatura en Matemáticas e Informática de la iversidad Francisco de Paula Santander?

Esta investigación muestra gran importancia ante el hecho de minimizar la repitencia de los estudiantes en el curso de la geometría analítica, que desde el punto de vista académico es una de las asignaturas de mayor rigor en el primer semestre de la licenciatura en matemáticas e informática de la universidad Francisco de Paula Santander. Y la idea es que el estudiante se mantenga en la universidad; de otro modo, con este tipo de investigaciones se pretende alimentar la línea de investigación en Educación Matemática del departamento de Matemáticas y Estadística de la UFPS.

\section{Metodología}

Se busco caracterizar los resultados observados en una población de individuos organizados en dos grupos, uno control y uno experimental; tomando en cuenta porcentajes de calificaciones, y otros resultados de evaluación, actividades realizadas, entre otros.

Para ello se elaboran los instrumentos necesarios para cada uno de los niveles o fases de aprendizaje según lo indica el modelo, y se realiza la evaluación con ítems que se relacionen con las características de cada uno de los niveles del mismo. Se tuvo en cuenta las habilidades generales para el aprendizaje, el nivel de implicación psicoafectiva en el proceso, el dominio del contenido en la asignatura, el avance en orden a lo largo de los niveles, el éxito en un nivel particular, quien aprende debe haber asimilado las estrategias de los niveles precedentes.

En el desarrollo de la presente investigación se ejecutaron varias etapas:

Primera etapa: (Diagnóstico) El proceso de investigación se inicio con la prueba diagnóstica para valorar nivel de razonamiento geométrico en los estudiantes de la muestra al iniciar el curso de Geometría Analítica. Se Tabularon los resultados de acuerdo a los aciertos y no aciertos en cada uno de los ítems.

A partir de los resultados de la prueba diagnóstica, se desarrollaran talleres teniendo en cuenta los niveles de razonamiento. De acuerdo con el modelo de van Hiele si el aprendiz es guiado por experiencias instruccionales adecuadas, avanza a través de los cinco niveles de razonamiento, empezando con el reconocimiento de figuras como todos (nivel 1), progresando hacia el descubrimiento de las propiedades de las figuras y hacia el razonamiento informal acerca de estas figuras y sus propiedades (niveles 2 y 3 ), y culminando con un estudio riguroso de geometría axiomática (niveles 4 y 5). El nivel 1 es denominado nivel de reconocimiento o visualización; el nivel 2, nivel de análisis; el nivel 3 clasificación o abstracción; el nivel 4 deducción, y el nivel 5 rigor. El modelo es recursivo, es decir cada nivel se construye sobre el anterior, concediéndose el desarrollo de los conceptos espaciales y geométricos como una secuencia desde planteamientos inductivos y cualitativos, hacia formas de razonamiento cada vez más deductivas y abstractas.

Segunda Etapa (Talleres): aplicación de la teoría Van Hiele en la clase de geometría del grupo experimental, los tópicos "Angulo entre rectas y distancia de un punto a una recta". Se diseñó pruebas teniendo en cuenta cada uno de los indicadores citados para medir las dimensiones de la variable de investigación. Se pudo utilizar algunas actividades que han 
formado parte en investigaciones anteriores. Se validó los instrumentos en opinión de docentes expertos en el área. Evaluación continúa de los logros alcanzados por los estudiantes durante la aplicación de los mismos. Pruebas parciales que contengan ítems con los niveles de razonamiento que propone Van Hiele. A su vez se realizaron entrevistas para valorar la metodología aplicada, y algunas implicaciones afectivas que pueden influenciar en el proceso. El grupo control tuvo las clases en forma tradicional. La entrevista tomó las respuestas dadas por el estudiante en cada tópico y se complementó con las razones que alude para cada razonamiento.

Tercera etapa: análisis de los resultados obtenidos por ambos grupos.

El diseño de investigación se centra en un Proceso Exploratorio bajo la modalidad de Campo, en el área de la aplicación y evaluación de modelos en la enseñanza. Asi mismo De Investigación Descriptiva y de De Investigación Explicativa.

\section{Resultados}

- Para efectos de tratamiento estadístico, se programo en una primera etapa, la aplicación de una prueba diagnóstica (Pre-test), para determinar el nivel de razonamiento en los estudiantes que inician el curso de Geometría Analítica.

- En una segunda etapa para el Grupo Experimental, se orientó, la aplicación del modelo siguiendo la teoría Van Hiele, desarrollando una serie de actividades debidamente organizadas en Guías didácticas de trabajo, orientadas por el docente. Para el Grupo Control se desarrollaron las actividades con un proceso tradicional de clase. Cada una de las actividades se evaluó en una prueba (Post-test).

- Al finalizar se hace un análisis comparativo para valorar el nivel de razonamiento geométrico en cada uno de los grupos Experimental y Control.

- Con la prueba diagnóstica se pretende de alguna forma, recopilar información personal como es el tiempo de egreso de los estudiantes y la edad, con el fin de caracterizar la población estudio, también se puede conocer el nivel socioeconómico del estudiante, al igual si su institución de egreso es de carácter Oficial o privada. Por otra parte y apoyados en los lineamientos curriculares y Estándares de Calidad, en sus Competencias establecidos por el Ministerio de Educación Nacional se busca valorar el Razonamiento Geométrico que traen los Estudiantes cuando finalizan su Escolaridad de la Secundaria e ingresan a la Universidad.

- Posiblemente el nivel socioeconómico y la institución de egreso que tiene el estudiante, si es de carácter oficial o privada, si podría influir en el rendimiento académico, pero en este caso los dos grupos están en con nivel socioeconómico homogéneo, específicamente en un estrato 3, al igual que la institución de egreso casi en la que en un $90 \%$ del total es de carácter oficial.

- En una segunda parte se busco conocer la cantidad de conocimiento geométrico que traen los estudiantes al iniciar el curso de Geometría Analítica. Los resultados se presentan de acuerdo a cada una de las preguntas de la prueba según indicadores establecidos.

- Los resultados según las medias por pregunta como Acertada, No Acertada o No responde, se muestra en los siguientes gráficos.

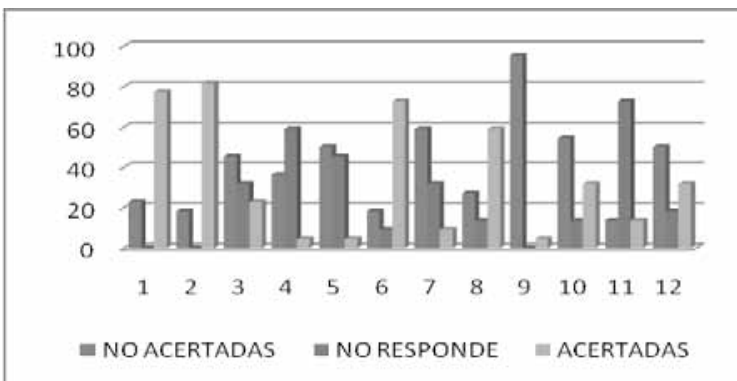

- Se observa en el gráfico que los estudiantes que conforman el Grupo Experimental, presentan en un gran número de preguntas con porcentaje alto de respuestas no acertadas.

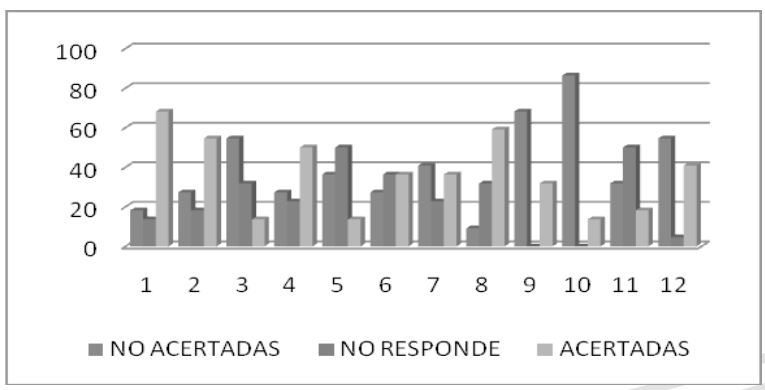


- Se observa en el gráfico que los estudiantes que conforman el Grupo control, presenta homogeneidad en sus respuestas acertadas y no acertadas.

- Se verifica que valoración total de la Prueba Diagnóstica para cada grupo. El Grupo Experimental y el Grupo Control obtuvieron resultados Deficientes, ya que la nota mínima aprobatoria es de 50 puntos en una escala de 0 a 100 puntos. La media del Grupo Control de 36,37 puntos, con La media del Grupo Experimental de 34,47 puntos.

- El grupo control tiene mayor porcentaje de estudiantes que responde satisfactoriamente a las competencias interpretativa y argumentativa, pero en situaciones propositivas el grupo experimental supero al grupo control. Por efectos del promedio en cada grupo se verifica mayor nivel de razonamiento o mayores conocimientos geométricos en el grupo control, concluyendo así que los porcentajes de respuestas acertadas en la Competencia interpretativa y argumentativa del grupo control, supero el porcentaje de respuestas acertadas en la competencia propositiva del grupo experimental.

- Cabe resaltar que según resultados individuales por pregunta en el grupo experimental y el grupo control no hay gran diferencia ya que en el caso de preguntas no acertadas el grupo experimental tuvo un $40,91 \%$, y el grupo control un 40,15\%, mientras que en la situación de no responde a la pregunta, el grupo control tiene un $23,48 \%$ y el grupo experimental un $24,62 \%$. Por lo tanto se concluye que dicha superación del grupo control sobre el grupo Experimental es muy poca.

- Aunque la diferencia de conocimientos geométricos del grupo control y del grupo experimental no es muy grande, se podría verificar si el año de egreso de los estudiantes influye en estos resultados, ya que el grupo control muestra mayor porcentaje de egreso el año anterior. Pero esta situación se deja a consideración de otro trabajo de investigación para ver que tanto influye el tiempo de egreso de los estudiantes en el nuevo conocimiento que adquieren al ingresar a la universidad, puesto que los preconceptos están mas cerca de recordarlos.

- En la segunda etapa de esta investigación se aplicó el modelo para cada uno de los niveles estudiados, permitiendo así que se valoren a partir de la actividad de los estudiantes.

- Tanto para el grupo Control como para el grupo experimental, al inicio de cada clase se informó el contenido a estudiar y los conocimientos previos con el fin de tomarlos como guía. Luego los estudiantes realizaban actividades dirigidas al descubrimiento y aprendizaje de los conceptos y propiedades fundamentales de dicho contenido.

- Al finalizar los estudiantes reflexionaban sobre los procedimientos, las dificultades y las soluciones encontradas, logrando enriquecer el conocimiento de cada individuo al detectar los métodos y resultados incorrectos para afianzar los correctos.

- La evaluación de los objetivos planteados en cada ítem de los instrumentos aplicados al grupo experimental, se realizo mediante preguntas en entrevista grupal e individual durante el desarrollo del mismo, a su vez se realizaron entrevistas a los estudiantes para determinar el nivel de aceptación psicoafectiva y la forma como estaban asimilando las diferentes actividades aplicadas, tomando en cuenta que serán futuros docentes que pudiesen poner en practica la misma.

- La actividad desarrollada con el grupo Experimental se género en un tiempo promedio de una hora por nivel, hasta el nivel 3. Para el nivel 4 se necesito casi las dos horas de intervención debido al rigor que exigía la actividad.

En cuanto a los resultados de la aplicación del modelo tenemos:

NIVEL 0: Visualización o reconocimiento. Un $68,18 \%$ del grupo, realizo las descripciones con el vocabulario geométrico adecuado, e identificaron elementos propios en cada figura presentada. En un $45,45 \%$ dieron respuesta acertada a la relación 
que tenían todas las imágenes a través de una reproducción gráfica, encontrando lo que se busco denotar específicamente en ellos. Los demás siguieron el proceso durante la clase, escuchando y participando hasta encontrarse al nivel de todos.

NIVEL 1: Análisis. En este nivel los estudiantes describieron y nombraron los elementos que caracterizaban las imágenes anteriores en un $59,09 \%$, realizaron las representaciones gráficas según las descripciones de los elementos y sus características en un $77,27 \%$, reconocieron las figuras mediante sus partes y propiedades estableciendo nuevas relaciones y afirmando las mismas, al igual que iniciaron un proceso de generalización en un $50 \%$, los demás durante los procesos iban identificando y dando respuestas acertadas de acuerdo a sus descubrimientos unos con ayuda de otros, hasta lograr alcanzar todos el mismo nivel.

NIVEL 2: Ordenación o clasificación. En este nivel señalaron las condiciones Necesarias y suficientes que deben cumplir en su razonamiento matemático en un 13,63\%, reconocen como unas propiedades se derivan de otras y las consecuencias de estas relaciones en un 45,45\%, siguen pasos individuales pero no asimilaron en forma global en un $36,36 \%$, siguieron la demostración inicial planteada en un 13,63\%. Los demás durante cada proceso para dar respuesta a las actividades desarrolladas, preguntaron, intervinieron aclararon dudas y realizaron procesos con seguimiento logrando al final alcanzar este nivel.

NIVEL 3: Deducción formal. En este nivel en un $18,18 \%$ comprendieron y manejaron relaciones entre propiedades empleando un razonamiento lógico formal para construir una demostración. Los demás a pesar de los diferentes procesos ya adquiridos demoraron pero alcanzaron el nivel orientados por el docente.

NIVEL 4: Rigor. A través del ejercicio práctico según el instrumento aplicado, en un 22,72\% alcanzaron este nivel no muy rápido pero si en menos tiempo que los demás, para lograr asimilarlo. Cuando finalizo la actividad rigurosa, los demás estudiantes alcanzaron dicho nivel con asesoría del docente y de sus mismos compañeros.
- Para la tercera etapa se aplico una prueba al final de la aplicación del modelo para el grupo Experimental y al final de la explicación del tema para el grupo control. La prueba duro 2 horas en tiempo de clase. Se aplico al total, es decir los 44 estudiantes matriculados en el curso, en el mismo tiempo y en la misma aula de clase. Se asumió como la valoración del logro del objetivo que se buscaba con dicho tema.

- Con esta prueba se buscaba que el estudiante solo con responder ciertas preguntas y resolver alguna situación desconocida sobre un tema no abordado en clase, llegara el solo a abordar dicho tema, realizando el proceso lógico para llegar a un nivel de razonamiento alto, sin la debida asesoría del docente.

- Se verifica que valoración total de la Prueba final para cada grupo. El Grupo Experimental y el Grupo Control obtuvieron resultados favorables, ya que en ambos grupos superaron el $50 \%$ de sus respuestas acertadas.

- El grupo con mejores resultados fue el grupo experimental por lo tanto las actividades aplicadas en este grupo fueron mas favorables y llevo a los estudiantes a mejores resultados. Por otra parte se afirma que el grupo experimental mejoró en los resultados obtenidos en la prueba diagnóstica después de la aplicación del modelo.

\section{Conclusiones}

- A medida que avanzó el semestre se noto un cambio positivo en la actitud de los estudiantes hacia el estudio de la Geometría Analítica, lo cual se evidenció en el interés y la motivación con la que desarrollaron las actividades propuestas. A su vez, manifestaron colocarla en practica en un futuro cuando se desempeñen como docentes en el área.

- Los resultados obtenidos durante los procesos desarrollados en las clases y en las pruebas evidenciaron que los grupos control y experimental inicialmente arrancaron con un nivel de razonamiento no muy bueno, y los 
resultados finales mejoraron y se noto que el grupo experimental supero al del grupo control debido a la metodología aplicada.

- Este modelo puede ser aplicado en cualquier tópico de conocimiento matemático ya que es flexible, garantizando así a los estudiantes una forma valida de aprender significativamente procesos geométricos para rendir de manera satisfactoria en la Geometría Analítica.

- Si un futuro docente en matemáticas logra aprender significativamente, es posible que su rendimiento como profesional sea eficiente llevando de manera directa a que el proceso de enseñanza tenga mayor credibilidad y así lograr que el proceso de aprendizaje de los estudiantes sea significativo.

- Con la investigación se evidenció claramente lo planteado por Fernando Fouz, Berritzegune de Donosti,en su paseo por la geometría, de que La idea básica de partida, dicho de forma sencilla y rápida, es que "el aprendizaje de la Geometría se hace pasando por unos determinados niveles de pensamiento y conocimiento", "que no van asociados a la edad" y "que sólo alcanzado un nivel se puede pasar al siguiente". Es más, se señala que cualquier persona, y ante un nuevo contenido geométrico a aprender, "pasa por todos esos niveles y, su mayor o menor dominio de la Geometría, influirá en que lo haga más o menos rápidamente".

- Todo docente de geometría debe tener presente el nivel de razonamiento geométrico en el que se encuentran los estudiantes antes de iniciar determinado tema abordar si se espera resultados significativos durante el aprendizaje del mismo.

- Cuando el docente conoce el nivel de razonamiento en el que se encuentran los estudiantes, debe organizar y planificar las actividades que permitan que el estudiante avance de un nivel a otro inmediatamente superior.

- Para la evaluación de los contenidos geométricos después de la aplicación del modelo van hiele se deben elaborar preguntas que se relaciones con cada uno de los diferentes niveles.
- El año de egreso de la secundaria que tiene el estudiante que inicia primer semestre en la educación superior influye significativamente en el logro de los objetivos planteados.

- La aplicación del modelo van hiele puede ser de gran utilidad desde el punto de vista didáctico, para el mejoramiento de las actividades docentes y a su vez el logro de los objetivos propuestos para determinado tema.

- Al evaluar la aplicación de este modelo, se verificó que es muy efectivo para elevar el nivel de razonamiento geométrico de los estudiantes durante el curso de la geometría analítica.

- El dialogo con los estudiantes dentro del proceso debe ser constante, ya que este permite evaluar el vocabulario geométrico adquirido.

- Con la aplicación del modelo se elevo el nivel de razonamiento geométrico en los estudiantes de primer semestre de la licenciatura en matemáticas e informática.

- La aplicación del modelo como una estrategia de aprendizaje sirvió de motivación para despertar el interés del estudiante en el estudio de la geometría analítica.

\section{Recomendaciones}

Se recomienda preparar a los estudiantes en conceptos básicos de geometría en un curso introductorio al ingresar al primer semestre de la universidad.

Se recomienda a los docentes prepararse en la utilización del modelo, como una nueva estrategia didáctica para alcanzar en los educando un nivel alto de razonamiento geométrico.

La relación psicoafectiva estudiante- docente influye en el aprendizaje de los educandos, por lo tanto se le recomienda a los docentes tener presente este aspecto para facilitar la adquisición del nuevo conocimiento a los educandos.

\section{Bibliografía}

CROWLEY, M. L. (1987): "The van Hiele model of development of geometric thought". En 
N.T.C.M.: Learning and teaching geometry, K12. N.T.C.M., Resten, pp. 1-16.

HERSCOVICS, N. y KIERAN, C. (eds.): Proceedings of the 11 th International Conference of the PME Vol, 3. Los autores (eds). Montreal. pp. 131-137.

GUTIERREZ, A. y JAIME,A. (1987): “Estudio de las características de los niveles de van Hiele".

GUTIERREZ, A. y JAIME, A. (1989): "Bibliografía sobre el modelo geométrico de van Hiele". Enseñanza de las Ciencias. vol nq 1, pp. 89-95.

GUTIERREZ, A. y JAIME, A. (1990): "Una propuesta de fundamentación para la enseñanza de la geometría: el modelo de van Hiele".

JAIME, A.; GUTIÉRREZ, A. (1990). Una propuesta de fundamentación para la enseñanza de la geometría: el modelo de van hiele. (Alfar: Sevilla), 295-384.(JaiGut90.pdf)

LOBO, Netsy. Aplicación del modelo propuesto en la teoría de van hiele para la enseñanza de la geometría. La Universidad del Zulia. Núcleo Punto Fijo. Dirección electrónica:http:// www.serbi.luz.edu.ve/scielo.php?script $=$ sci_ arttext\&pid $=$ S1317-22552004001000004\&lng= es\&nrm=iso)

HERNÁNDEZ, J. (1978): La enseñanza de las matemáticas modernas. Alianza Universidad. Madrid. HOFFER,A. (1983): "Van Hiele based reserach". 